\title{
The protective effects of vasopressin on experimental testicular torsion-detorsion in male rats
}

\author{
Amin Hasanvand ${ }^{1} \mathbb{D}$, Fariba Ahmadizar ${ }^{2}$, Sadegh Ebrahimi-Ghalaei ${ }^{3}$, Fatemeh Beyranvand ${ }^{4}$, Reza \\ Mohammadrezaei Khorramabadi ${ }^{3}$, Peyman Amanolahi Baharvand ${ }^{4}$, Abolfazl Abbaszadeh ${ }^{*}$ (D)
}

${ }^{1}$ Nutritional Health Research Center, Department of Pharmacology and toxicology, Faculty of Pharmacy, Lorestan University of Medical Sciences, Khorramabad, Iran

${ }^{2}$ Department of Epidemiology, Erasmus University Medical Center, Rotterdam, the Netherlands

${ }^{3}$ Student Research Committee, Lorestan University of Medical Sciences, Khorramabad, Iran

${ }^{4}$ Razi Herbal Medicines Research Center, Lorestan University of Medical Sciences, Khorramabad, Iran

${ }^{5}$ Department of Surgery, Lorestan University of Medical Sciences, Khorramabad, Iran

\section{AR T I C L E IN F O}

Article Type:

Original

\section{Article History:}

Received: 7 October 2018

Accepted: 10 December 2018

ePublished: 6 January 2019

\section{Keywords:}

Ischemia-reperfusion

Testicular rotation

Testis

Glutathione

Reactive oxygen species

Ischemia-reperfusion

\begin{abstract}
A B S T R A C T
Introduction: Vasopressin is a potent vasoconstrictor. Vasopressin can reduce damages caused by ischemia-reperfusion.

Objectives: The aim of this study was to evaluate the reducing effect of vasopressin on the damage caused by testicular torsion-detorsion in male rats.

Materials and Methods: Fifty male rats were used in this study. The rats were divided randomly into five groups (10 per group) as follows; 1) control group (without surgery), 2) sham group (surgery without torsion-detorsion), 3) torsion-detorsion surgery without any treatment, 4) torsion-detorsion surgery with vasopressin treatment $(0.1 \mu \mathrm{g} / \mathrm{kg}), 5)$ torsion-detorsion surgery with vasopressin treatment $(0.2 \mu \mathrm{g} / \mathrm{kg})$. After that, the rats were killed and subsequently the biochemical parameters were measured by commercial kits. Histological changes were assessed by H\&E staining.

Results: Using Kruskal Wallis test, we observed that the changes in the tissues of seminiferous tubes, testosterone levels, glutathione (GSH) and thioredoxin enzymes in the studied groups (control, sham, torsion-detorsion, torsion-detorsion with $0.1 \mu \mathrm{g} / \mathrm{kg}$, torsion-detorsion with $0.2 \mu \mathrm{g} / \mathrm{kg}$ ) were statistically significant.

Conclusion: Intracellular mechanisms of vasopressin involved in the regulation of protective mechanism and can reduce damages caused by ischemia-reperfusion. Vasopressin improves the process of changes in the testicular seminiferous tubes of rats with torsion-detorsion. Moreover, it also increases serum levels of testosterone, GSH and thioredoxin enzymes compared to the torsiondetorsion group.
\end{abstract}

Implication for health policy/practice/research/medical education:

Testicular torsion is an urgent condition in pediatric urology. Testicular torsion refers to testicular rotation around its vascular axis (spermatic cord), which results in obstruction and stoppage of blood flow and eventually the loss of living tissue (infarction). Ischemic condition causes damage to the testes due to the blood flow disruption. Furthermore, reperfusion of blood flow after ischemia (detorsion) can exacerbate the damage to the testis. Vasopressin as an antidiuretic hormone (ADH), is a pituitary hormone found in most mammals including humans. It is mainly made in supra-optic nuclei. Vasopressin is a polypeptide hormone with nine amino acids and is similar to the oxytocin hormone. In an experimental study, we found that vasopressin possesses intracellular mechanisms similar to the protective preconditioning mechanism which can reduce damages caused by ischemia-reperfusion.

Please cite this paper as: Hasanvand A, Ahmadizar F, Ebrahimi-Ghalaei S, Beyranvand F, Mohammadrezaei Khorramabadi $\mathrm{R}$, Amanolahi Baharvand $\mathrm{P}$, et al. The protective effects of vasopressin on experimental testicular torsion-detorsion in male rats.

J Nephropharmacol. 2019;8(1):e10. DOI: 10.15171/npj.2019.10

\section{Introduction}

The testicular torsion refers to testicular rotation around its vascular axis (spermatic cord), which results in obstruction and stoppage of blood flow and eventually the loss of living tissue (infarction) (1). Therefore, this is a real surgical emergency, which leads to irreversible damage to the testicular tissue after 4 hours of spermatic cord closure. This condition occurs predominantly during puberty and between the ages of 12 to 20 . The statistics concerning the effect of testicular torsion on young men 
show that one in 4000 suffer from testicular torsion (2). Puberty and some diseases including tumors of testicle, testicles with horizontal lie and cryptorchidism contribute to the development of testicular torsion (3). Testicular torsion with $360^{\circ}$ can lead to complete obstruction of vessels. This process can ultimately cause ischemia, especially 24 hours after the beginning of torsion (4). Ischemic condition causes damage to testis because of the disruption of blood flow. Furthermore, reperfusion of blood flow after ischemia (detorsion) can exacerbate the damage to testis. A series of possible underlying events including oxidative stress, particularly reactive oxygen species (ROS), inflammatory cells and cytokines, lipid peroxidation, apoptosis, anoxia, which finally could cause infertility, play roles in the damage caused by testicular torsion-detorsion $(5,6)$. ROS generation is influential in the damage induced by ischemia which leads to cellular injuries (7). They also activate inflammation via increased recruitment of leukocytes and pro-inflammatory cytokines levels. Subsequently, ROS generation and inflammation induce apoptosis during testicular ischemia/reperfusion injury $(8,9)$. On the other hand, hypoxia is proposed as a trigger factor which induces damage pathway via increased inflammation and oxidative stress (10). Various treatments were used to attenuate testicular ischemiareperfusion in a rat model. In fact, a variety of drugs and compounds such as phosphodiesterase inhibitors, vitamins, selenium, plant extracts and other antioxidants are used for this purpose (11).

Vasopressin, or antidiuretic hormone $(\mathrm{ADH})$, is a pituitary hormone found in most mammals including humans. It is mainly made in supra-optic nuclei. Vasopressin is a polypeptide hormone with nine amino acids and is similar to the oxytocin hormone. This molecular similarity justifies their relative functional similarity (12). The most important function of this hormone is to maintain the balance of water in the body. While low concentrations of vasopressin increase the water retention by the kidneys, its higher concentrations have potent contractile effects on the entire cellular arteries resulting in increased arterial pressure (13). Vasopressin receptors are divided into four groups (V1, V2, V3 and OTRs) among which V1 is present in testis. It brings about many neurological effects on the brain. Likewise, it is associated with mating behaviors in a variety of rats. Several studies have been carried out on various biological effects of vasopressin (14). However, no study has been done so far on the effect of vasopressin on the damage caused by the testicular torsion-detorsion. Since ischemiareperfusion in the testicle, like ischemia-reperfusion in other organs such as the brain, heart and kidneys, causes oxidative stress and inflammation.

\section{Objectives}

In the present study, we aimed to investigate the effect of vasopressin on testicular damage caused by ischemia- reperfusion in rats.

\section{Materials and Methods}

Chemicals

The following chemicals were purchased from Sigma Aldrich Company (USA) and Merck Company (Germany).

\section{Study design}

Fifty Sprague-Dawley male rats (weighing 200-220 g) were obtained from Razi herbal medicines research center, Lorestan, Iran. After preparation, the rats were housed in an animal laboratory for 1 week. Standard access to food and water was prepared for them. Moreover, the standardization of laboratory conditions was carried out for them including temperature of $22^{\circ} \mathrm{C}$ and 12 -hour light and 12-hour dark cycle. Then, they were divided randomly into five groups as follows:

1. Group 1; control group (healthy); no surgery was performed.

2. Group 2; sham group; this group got surgery without torsion-detorsion (ischemia-reperfusion).

3. Group 3; this group got surgery together with torsion-detorsion (ischemia-reperfusion). They did not receive any treatment either.

4. Group 4; this group got surgery together with torsiondetorsion (ischemia-reperfusion). They received vasopressin $(0.1 \mu \mathrm{g} / \mathrm{kg})$, too.

5. Group 5; this group got surgery together with torsiondetorsion (ischemia-reperfusion). They received vasopressin $(0.2 \mu \mathrm{g} / \mathrm{kg})$, too.

In order to perform anesthesia, ketamine $(50 \mu \mathrm{g} / \mathrm{kg})$ and xylazine $(5 \mu \mathrm{g} / \mathrm{kg})$ were used in intraperitoneal injection.

\section{Surgical procedure}

Torsion-detorsion (ischemia-reperfusion) was conducted using 720-degree twisting of testis for 90 minutes according to previous researches. This degree of twisting is necessary to disrupt the seminal epithelium. Subsequently, vasopressin injections were performed at the doses of 0.1 and $0.2 \mu \mathrm{g} / \mathrm{kg} 10$ minutes before detorsion.

\section{Histological evaluation of seminiferous tubules}

All animals were killed by spinal cord movement after the treatment period. Then, their testicles were removed from the abdominal cavity. The testes were immersed in Bouin's fixative solution for 72 hours at room temperature to achieve tissue fixation. Three days after fixation, the specimens were processed. Subsequently, tissue samples were cut into $5 \mu \mathrm{m}$ thickness, and then they were stained with hematoxylin and eosin for the purpose of microscopic evaluation. Johnsen's method was used to study the maturity and quality of seminiferous tubules.

\section{Hormonal measurement}

Blood samples were prepared from hearts of rats for the purpose of hormonal measurement. Then, serum was 
separated by centrifuging the blood at $3000 \mathrm{rpm}$ for 15 minutes. Finally, the level of testosterone was measured by ELISA kit.

\section{Thioredoxin and glutathione measurement}

These parameters were estimated by the use of ELISA kit (15).

\section{Ethical issues}

The protocol of this study is designed in accordance with the ethical principles of the International Committees for the Protection of Animal Rights Laboratory. This project was approved by the Ethics Committee of Lorestan University of Medical Sciences.

\section{Statistical analysis}

Appropriate central and diffusion indices were calculated for data analysis using one-way ANOVA or Kruskal-Wallis test. SPSS version 16 was also used for data analysis. The significance level was considered as $P<0.05$. Hormonal and enzymatic data were analyzed using GraphPad

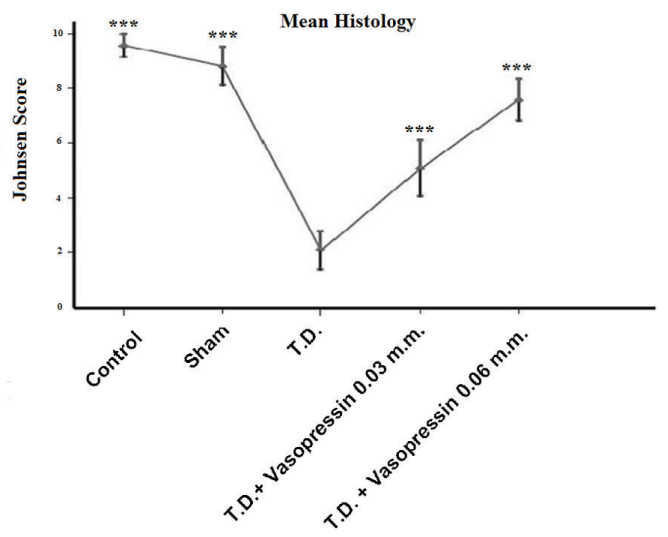

Figure 1. Comparison of histopathologic changes of testicular tissue in different groups. ${ }^{* *}$ Significant difference compared to the torsion -detorsion group at the level $P<0.001$. software and one-way ANOVA data.

Results

Comparison of testicular tissue changes in treatment groups

Based on the results of one-way ANOVA, the difference between the mean histopathological changes of the testes in different study groups (control, sham, vasopressin at dose of $0.1 \mu \mathrm{g} / \mathrm{kg}$, vasopressin at dose of $0.2 \mu \mathrm{g} / \mathrm{kg}$ ) compared to the torsion -detorsion group, was statistically significant at the level $P<0.001$ (Figure 1). As shown in the diagrams and figures, the improvement in the process of tissue changes in seminiferous tubules following testicular torsion was significantly distinct in both vasopressin receiving groups compared to the torsion-detorsion group (Figure 2).

\section{Comparison of testosterone levels in treated groups}

Based on the results of one-way ANOVA, the difference in mean of changes in testosterone levels in the vasopressin treated group $(0.2 \mu \mathrm{g} / \mathrm{kg})$ compared to the torsion -detorsion group was statistically significant $(P<0.05)$. The control group and the sham group were statistically significant in comparison with the torsion-detorsion group $(P<0.001)$ (Figure 3$)$.

Based on the results of one-way ANOVA, the difference in mean of changes in glutathione (GSH) levels in the vasopressin treated group $(0.2 \mu \mathrm{g} / \mathrm{kg})$ compared to the torsion-detorsion group was statistically significant $(P<0.05)$. The control group and the sham group were statistically significant in comparison with the torsiondetorsion group $(P<0.001)$ (Figure 4$)$.

\section{Comparison of thioredoxin levels in treated groups}

Based on the results of one-way ANOVA, the difference in mean of changes in thioredoxin levels in the vasopressin treated group $(0.1 \mu \mathrm{g} / \mathrm{kg})$ compared to the torsiondetorsion group was statistically significant $(P<0.05)$. Moreover, the difference in mean of changes in thioredoxin

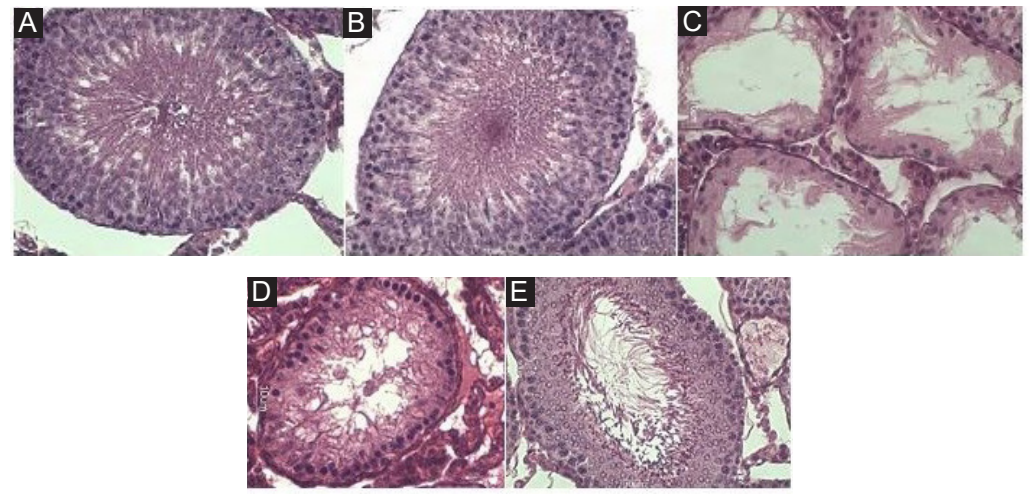

Figure 2. Tissue changes of seminiferous tubules in different groups using H\&E staining. A) Healthy group, B) Sham group, C) Control group, D) Vasopressin treated $(0.1 \mu \mathrm{g} / \mathrm{kg})$ group, E) Vasopressin treated $(0.2 \mu \mathrm{g} / \mathrm{kg})$ group. 
Testosterone values

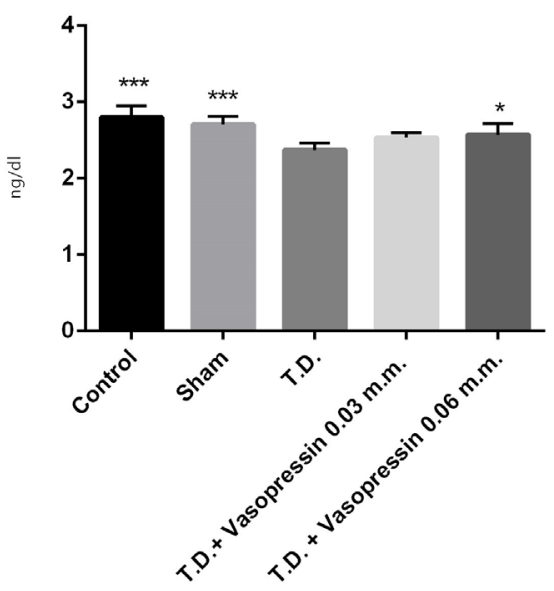

Figure 3. The comparison of changes in testosterone levels in different groups. * Significant difference compared to the torsion -detorsion group at the level $P<0.05$. ${ }^{* * *}$ Significant difference compared to the torsion-detorsion group at the level $P<0.001$.

Glutathione (GSH) concentration

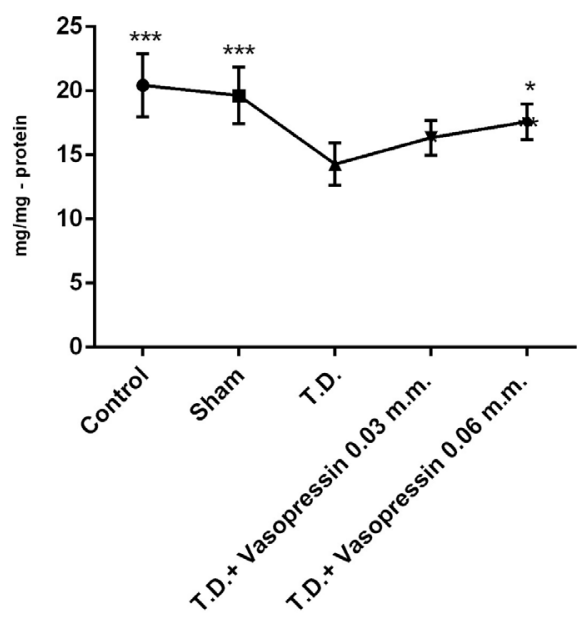

Figure 4. The comparison of changes in GSH levels in different groups. * Significant difference compared to the torsion -detorsion group at the level $P<0.05$. ${ }^{* * *}$ Significant difference compared to the torsion-detorsion group at the level $P<0.001$.

levels in the vasopressin treated group $(0.2 \mu \mathrm{g} / \mathrm{kg})$ compared to the torsion -detorsion group was statistically significant $(P<0.05)$. The control group and the sham group were statistically significant in comparison with the torsion-detorsion group $(P<0.001)$ (Figure 5).

\section{Discussion}

Testicular torsion-detorsion can lead to infertility in severe cases. Complex mechanisms are associated with pathogenesis of testicular ischemia-reperfusion. ROS generation and inflammatory responses are the most important underlying factors which could develop
Thioredoxin level

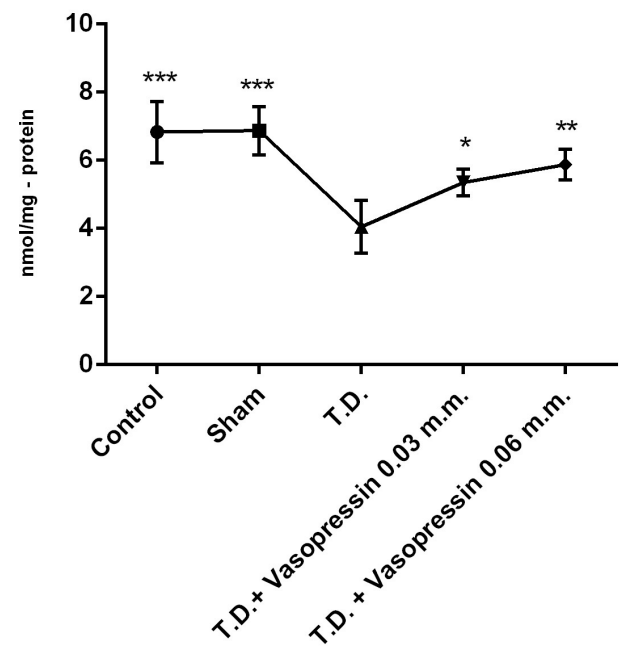

Figure 5. The comparison of changes in thioredoxin levels in different groups. * Significant difference compared to the torsion -detorsion group at the level $P<0.05$. ${ }^{* *}$ Significant difference compared to the torsion -detorsion group at the level $P<0.01$. ${ }^{* * *}$ Significant difference compared to the torsion -detorsion group at the level $P<0.001$.

testicular injury. Various kinds of treatments could be utilized to ameliorate testicular torsion-detorsion (11). Vasopressin, for instance, has been used for the treatment of cardiac ischemia-reperfusion injury. Because of the existence of similar mechanisms associated with the development of different types of ischemia-reperfusion, we decided to evaluate the ameliorative effects of vasopressin on testicular torsion-detorsion. The results of our study in the field of histology showed that maturity and quality of seminiferous tubules improved by administration of vasopressin. Furthermore, the biochemical results of our research indicated that serum levels of testosterone, GSH and thioredoxin were modified because of administration of vasopressin.

A wide range of compounds, including drugs, could be used to treat testicular torsion-detorsion. A similar study indicated that phosphodiesterase inhibitors such as vardenafil, sildenafil, trapidil, and dipyridamole could attenuate testicular ischemia-reperfusion injury. It is established that vardenafil is an efficient drug against oxidative stress caused by testicular torsion-detorsion as it reduces malondialdehyde (MDA) levels. However, it cannot ameliorate histological changes in testes. Sildenafil, as another phosphodiesterase inhibitor, exhibits antioxidant properties via increased levels of GSH, catalase and glutathione peroxidase. It also shows beneficial effects on improving histopathological parameters caused by torsion. Other phosphodiesterase inhibitors, like trapidil and dipyridamole, decreased histological damage caused by testicular torsion (16-18). Since vitamin $\mathrm{C}$ is a potent antioxidant, it reduces the damage 
caused by testicular torsion. Vitamin $\mathrm{E}$ is also similar to vitamin $C$ with regard to its capacity to prevent testicular damage. Dexpanthenol is proposed as an anti-atrophy agent against testicular injury (19,20). Alpha-lipoic acid and selenium play their roles via increasing antioxidant defect particularly GSH in testicular injury (21). It has been reported that different types of hormones such as erythropoietin, melatonin and dehydroepiandrosterone (DHEA) are influential in reducing the damage caused by testicular ischemia-reperfusion. The antioxidant and anti-inflammatory effects of erythropoietin have been documented previously. Hence, erythropoietin shows its therapeutic effect via decreasing inflammation (22). Melatonin, another effective hormone in the treatment of testicular torsion, recovers histopathological changes through increasing Johnsen's score and decreasing abnormal sperms (23). Dehydroepiandrosterone reduces apoptosis of spermatocytes (24). Plant-derived active ingredients are considered as successful therapeutic strategies against different types of ischemia-reperfusion injuries, especially testicular ones. Ternatin, quercetin, lycopene and thymoquinone are some of these compounds which can be used in the treatment of testicular ischemiareperfusion injury. For instance, ternatin affects oxidative stress by its antioxidant property (25). Moreover, quercetin affects histopathological changes via increasing mean seminiferous tubule diameter and Johnsen's score (26). Lycopene is also known for its potent antioxidant property. It is found in tomato. The possible mechanism, which is assumed for its protective effect on testicular damage, is related to its activity against oxidative stress in testes (27). Thymoquinone and l-carnitine exhibit antioxidant property via decreasing MDA level in testes (28). Previous investigations have confirmed the antioxidant effects of plants such as Panax ginseng, garlic and Ginkgo biloba. These plants have been used in the treatment of testicular ischemia-reperfusion as they can decrease oxidative stress (11).

All of the above studies confirm our results. In fact, most of these compounds were effective against the damage caused by testicular ischemia-reperfusion. Their therapeutic effects are generally exhibited by reducing oxidative stress similar to our study.

\section{Conclusion}

All in all, our research indicated that administration of vasopressin could decrease histopathological changes and oxidative stress. Hence, it is assumed that vasopressin can be proposed as an efficient choice to be used in the treatment of various types of ischemia-reperfusion injury.

\section{Acknowledgments}

The authors would like to thank Lorestan University of Medical Sciences, Khorramabad, Iran, because of its financial support. The authors also thank the head and staff of the Razi Herbal Medicines Research Center of
Lorestan University of Medical Sciences.

\section{Authors' contribution}

$\mathrm{AH}, \mathrm{FA}, \mathrm{SE}, \mathrm{FB}, \mathrm{RMK}$, and PAB conducted the research. $\mathrm{AH}$ and AA designed and supervised the study, analyzed the data and prepared the final draft of the manuscript. All authors read and signed the final paper.

\section{Ethical considerations}

Ethical issues (including plagiarism, data fabrication, double publication) have been completely observed by the authors.

\section{Conflict of interest}

The authors declare that they have no conflict of interest.

\section{Funding/Support}

This study was supported by Lorestan University of Medical Sciences with (Grant \# A-10-1758-6).

\section{References}

1. Ringdahl E, Teague L. Testicular torsion. Am Fam Physician. 2006;74.

2. Cuckow P, Frank J. Torsion of the testis. BJU Int. 2000;86:349-53.

3. Arap MA, Vicentini FC, Cocuzza M, Hallak J, Athayde K, Lucon AM, et al. Late hormonal levels, semen parameters, and presence of antisperm antibodies in patients treated for testicular torsion. J Androl. 2007;28:528-32.

4. Sharp VJ, Kieran K, Arlen AM. Testicular torsion: diagnosis, evaluation, and management. Am Fam Physician. 2013;15;88:835-840.

5. Lysiak JJ, Turner SD, Nguyen QA, Singbartl K, Ley K, Turner TT. Essential role of neutrophils in germ cellspecific apoptosis following ischemia/reperfusion injury of the mouse testis. Biol Reprod. 2001;65:718-25.

6. Turner TT, Bang HJ, Lysiak JL. The molecular pathology of experimental testicular torsion suggests adjunct therapy to surgical repair. J Urol. 2004;172:2574-8.

7. Reilly PM, Schiller HJ, Bulkley GB. Pharmacologic approach to tissue injury mediated by free radicals and other reactive oxygen metabolites. Am J Surg. 1991;161:488-503.

8. Al-Maghrebi M, Renno WM, Al-Ajmi N. Epigallocatechin3-gallate inhibits apoptosis and protects testicular seminiferous tubules from ischemia/reperfusioninduced inflammation. Biochem Biophys Res Commun. 2012;6;420:434-9. doi: 10.1016/j.bbrc.2012.03.013.

9. Isikdemir F, Kurcer Z, Dengiz G, Sipahi E, Banoglu Z, Baba $F$, et al. Effects of montelukast and zileuton on testicular torsion/detorsion injury in rats. Andrologia. 2014;46:59-64. doi: 10.1111/and.12042.

10. Reyes JG, Farias JG, Henríquez-Olavarrieta S, Madrid E, Parraga M, Zepeda AB, et al. The hypoxic testicle: physiology and pathophysiology. Oxid Med Cell Longev. 2012;2012:929285. doi: 10.1155/2012/929285.

11. Karaguzel E, Kadihasanoglu M, Kutlu O. Mechanisms of testicular torsion and potential protective agents. Nat Rev Urol. 2014;11:391-9. doi: 10.1038/nrurol.2014.135.

12. Baribeau DA, Anagnostou E. Oxytocin and vasopressin: 
linking pituitary neuropeptides and their receptors to social neurocircuits. Front Neurosci. 2015;24;9:335. doi: 10.3389/ fnins.2015.00335.

13. Wadei HM, Textor SC. The role of the kidney in regulating arterial blood pressure. Nat Rev Nephrol. 2012;8:602-9. doi: 10.1038/nrneph.2012.191.

14. Zelena D. Vasopressin in health and disease with a focus on affective disorders. Cent Nerv Syst Agents Med Chem. 2012;12:286-303.

15. Sadeghi A, Kalantar M, Molavinia S, Houshmand G, Bahadoram M, Esmaeilizadeh $M$, et al. Ameliorative effects of hydroalcoholic extract of Lavandula officinalis L. on cyclophosphamide-induced nephrotoxicity in mice. J Nephropathol. 2017;6:324-332. doi: 10.15171/jnp.2017.52.

16. Özgür BC, Telli O, Yuceturk CN, Sarici H, Ozer E, Surer $\mathrm{H}$, et al. The effect of sildenafil and udenafil on testicular damage following ischemia-reperfusion injury in rats. J Urol. 2014;192:1272-7. doi: 10.1016/j.juro.2014.04.011.

17. Tuglu D, Yuvanc E, Ozan T, Bal F, Yilmaz E, Atasoy P, et al. Protective effects of udenafil citrate, piracetam and dexmedetomidine treatment on testicular torsion/ detorsion-induced ischaemia/reperfusion injury in rats. Andrologia. 2016;48:676-82. doi: 10.1111/and.12499.

18. Abdel-Rahman MM, Mahmoud AM, Mostafa NM, Saleh ME, Eissa HM. Sildenafil reduces ischemia/reperfusion testicular injury after torsion/detorsion in rats. J Appl Pharm Sci. 2016;6:135-140.

19. Moghimian M, Soltani M, Abtahi H, Shokoohi M. Effect of vitamin $\mathrm{C}$ on tissue damage and oxidative stress following tunica vaginalis flap coverage after testicular torsion. J Pediatr Surg. 2017;52:1651-5. doi: 10.1016/j. jpedsurg.2017.07.001.

20. Romeo C, Antonuccio P, Esposito M, Marini H, Impellizzeri $\mathrm{P}$, Turiaco N, et al. Raxofelast, a hydrophilic vitamin E-like antioxidant, reduces testicular ischemia-reperfusion injury. Urol Res. 2004;32:367-71.

21. Ozbal S, Ergur BU, Erbil G, Tekmen I, Bagriyanık A, Cavdar $Z$. The effects of $\alpha$-lipoic acid against testicular ischemiareperfusion injury in rats. ScientificWorldJournal. 2012;2012:489248. doi: 10.1100/2012/489248.

22. Yazihan N, Ataoglu H, Koku N, Erdemli E, Sargin AK. Protective role of erythropoietin during testicular torsion of the rats. World J Urol. 2007;25:531-6.

23. Koksal M, Oğuz E, Baba F, Eren MA, Ciftci H, Demir M, et al. Effects of melatonin on testis histology, oxidative stress and spermatogenesis after experimental testis ischemia-reperfusion in rats. Eur Rev Med Pharmacol Sci. 2012;16:582-8.

24. Yapanoglu T, Aksoy Y, Gursan N, Ozbey I, Ziypak T, Calik M. Antiapoptotic effects of dehydroepiandrosterone on testicular torsion/detorsion in rats. Andrologia. 2008;40:3843. doi: 10.1111/j.1439-0272.2008.00806.x.

25. Guimarães SB, Santos JMV, Aragão AA, Kimura OS, Silveira ER, Vasconcelos PRLd. Ternatin pretreatment attenuates testicular injury induced by torsion/detorsion in Wistar rats. Acta Cir Bras. 2011;26:325-8.

26. Aktoz T, Kanter M, Aktas C. Protective effects of quercetin on testicular torsion/detorsion-induced ischaemiareperfusion injury in rats. Andrologia. 2010;42:376-83. doi: 10.1111/j.1439-0272.2010.01044.x.

27. Hekimoglu A, Kurcer Z, Aral F, Baba F, Sahna E, Atessahin A. Lycopene, an antioxidant carotenoid, attenuates testicular injury caused by ischemia/reperfusion in rats. Tohoku J Exp Med. 2009;218:141-7.

28. Gökçe A, Oktar S, Koc A, Gonenci R, Yalcinkaya F, Yonden Z, et al. Protective effect of thymoquinone in experimental testicular torsion. Urol Int. 2010;85:461-5. doi: $\quad 10.1159 / 000318890$.

Copyright $\odot 2019$ The Author(s); Published by Society of Diabetic Nephropathy Prevention. This is an open-access article distributed under the terms of the Creative Commons Attribution License (http://creativecommons.org/licenses/by/4.0), which permits unrestricted use, distribution, and reproduction in any medium, provided the original work is properly cited. 